\section{SAT0057 \\ THE EFFECT OF EXOSOMES FROM BONE MARROW MESENCHYMAL STEM CELLS ON OSTEOARTHRITIS}

H. Zhu, J. Ji, T. Fu, J. Yang, Z. Gu. Department of Rheumatology, Affiliated Hospital of Nantong University, Nantong, China

Background: Mesenchymal stem cells (MSCs) exert chondroprotective effects in clinical models of osteoarthritis (OA), but the exact mechanisms were still unclear. Exosomes that serve as carriers of genetic information have been implicated in many diseases and are known to participate in many physiological processes.

Objectives: Here, we investigate the therapeutic potential of exosomes from human bone marrow MSCs in alleviating OA and explore the mechanism.

Methods: Exosomes were harvested from conditioned culture media of BMMSCs by a sequential centrifugation process. The anterior cruciate ligament transection and destabilisation of the medial meniscus (DMM) surgery were performed on the knee joints of SD female rat as an OA model. After four weeks, the animals were followed by intra-articular injection of either BM-MSCs or their exosomes every week for four weeks. Cartilage destruction, matrix degradation and subchondral bone changes were evaluated with histological staining and microCT at the post-surgery 8 weeks. Primary human chondrocytes treated with IL-1 $\beta$ were used as an in vitro model to evaluate the effects of exosomes for 24 hours. Results: We found that intra-articular injection of BM-MSCs and BM-MSCs derived exosomes improve cartilage destruction and subchondral bone remodelling in ACLT+DMM model. BM-MSCs and exosomes equally protected rat from joint damage. These exosomes maintained the chondrocyte matrix by increasing collagen type II synthesis and decreasing ADAMTS5, MMP13 and Col II expression in the presence of $\mathrm{IL}-1 \beta$ in vitro. In addition, BM-MSCs derived exosomes were also shown to protect chondrocytes from apoptosis and senescence.

Conclusions: The exosomes from BM-MSCs exert a beneficial therapeutic effect on $\mathrm{OA}$ by not only repairing the degenerative cartilage, but also improving subchondral bone remodelling, which in turn provides a new target for OA drug and drug-delivery system development.

\section{REFERENCES :}

[1] Trounson A, McDonald C. Stem Cell Therapies in Clinical Trials: Progress and Challenges. Cell stem cell 2015;17:11-22.

[2] Stoorvogel W, Kleijmeer MJ, Geuze HJ, Raposo G. The biogenesis and functions of exosomes. Traffic 2002;3:321-30.

[3] Lai RC, Yeo RW, Lim SK. Mesenchymal stem cell exosomes. Seminars in cell \& developmental biology 2015;40:82-8.

[4] Zhang S, Chu WC, Lai RC, Lim SK, Hui JH, Toh WS. Exosomes derived from human embryonic mesenchymal stem cells promote osteochondral regeneration. Osteoarthritis and cartilage 2016;24:2135-40.

[5] Wang Y, Yu D, Liu Z, et al. Exosomes from embryonic mesenchymal stem cells alleviate osteoarthritis through balancing synthesis and degradation of cartilage extracellular matrix. Stem cell research \& therapy 2017;8:189.

Acknowledgements: No.

Disclosure of Interest: None declared

DOI: 10.1136/annrheumdis-2018-eular.6112

\section{SAT0058 ADALIMUMAB:TNF COMPLEXES ARE CLEARED MORE EFFICIENTLY BY HUMAN OSTEOCLASTS THAN THOSE WITH ETANERCEPT THROUGH FCG-RECEPTOR BINDING AND INTERNALISATION}

B.P. Harvey, J. Cohen-Solal, Z. Kaymakcalan. Biologics, Abbvie Bioresearch Center, Worcester, USA

Background: TNF-alpha (TNFa) has been shown to contribute to osteoclastogenesis (OCgenesis) independently and in conjunction with M-CSF or RANKL, two key cytokines involved in osteoclast $(\mathrm{OC})$ development. We have previously demonstrated that TNF enhances the kinetics of RANKL-induced human OCgenesis and that its effects are mitigated more effectively by the anti-TNF biologic adalimumab (ADA) as compared to etanercept (ETN).

Objectives: To determine whether Fc-gamma receptor (FcgR)-mediated internalisation of the biologic:TNF complexes is a contributing mechanism responsible for the difference in effectiveness between ADA and ETN in preventing TNFenhanced OCgenesis.

Methods: TNF biologics [ADA and ETN] alone or in preformed complexes with TNFa at 50:1 molar ratio were tested for FcgR binding by flow cytometry using CHO stably transfected with human FCGRs (FcgRI, FcgRIIA, -RIIB, -RIIC FcgRIIIA and -RIIIB). FcgR expression and binding of preformed biologic:TNF complexes at 10:1 ratio $\pm F c g R$ blocking antibodies to primary human OC precursors (OCP) was evaluated by flow cytometry. FcgR-mediated internalisation was assessed by monitoring a reduction in OC survival in response to preformed biologic:TNF complexes (25:1 ratio) bound with saporin (ZAP), a ribosome-inactivating toxin, as anti-human Fc IgG Fab conjugate $\pm F c g R$ blocking antibodies

Results: The binding study to $\mathrm{CHO}$ (human FcgRs) cell lines showed that monomeric ADA and ETN bind similarly to FcgR (highly on high affinity FcgR and loosely on low affinity FcgRs) while preformed biologics: TNF complexes bind differently. ADA:TNF complexes bind to low affinity FcgR, whereas ETN:TNF keep a monomeric binding profile with no gain of binding to low affinity FcgR. OCP were found to express mostly FcgRII early in development with predominant binding of only ADA:TNF, not ETN:TNF, to this FcgR with additional binding to undefined receptor(s). Despite subsequent increases in FcgRI and RIII later on, ADA:TNF still preferentially bound to FcgRII on the matured OCP with minimal binding to RIII, whereas ETN:TNF binding was observed only to FcgRI. Exposure of OCP to ADA:TNF:ZAP(toxin) complexes led to a significant reduction (4-fold) in mature OC due to complex internalisation as compared to human lgG:ZAP +TNF conditions that was partially rescued only with the addition of FcgRII blocking antibody. Interestingly, a 1.5-fold reduction in mature OC was observed with ETN:TNF ZAP

Conclusions: Our in vitro findings demonstrate that human OCP can bind and internalise ADA:TNF complexes more efficiently than ETN:TNF complexes. In addition, this process is partially mediated through FcgRII. Clearance of the ADA TNF complexes may help reduce exposure of the OCP to localised TNF by removing TNF more effectively in the joint environment. Additional in vivo analysis need to be done to verify these in vitro findings.

Acknowledgements: Authors thank Drs. Jochen Salfeld, Dhaval Nanavati and Yonghao Cao for their critical review of the science and publication.

Disclosure of Interest: B. Harvey Shareholder of: AbbVie Inc., Employee of: AbbVie Inc., J. Cohen-Solal Shareholder of: AbbVie Inc., Employee of: AbbVie Inc., Z. Kaymakcalan Shareholder of: AbbVie Inc., Employee of: AbbVie Inc. DOI: 10.1136/annrheumdis-2018-eular.3804

\section{SAT0059 IMPAIRMENT IN HYDROGEN SULFIDE SYNTHESIS IN OSTEOARTHRITIC CHONDROCYTES FROM DIABETIC PATIENT AND UNDER A HIGH GLUCOSE STRESS}

C. Vaamonde-Garcia ${ }^{1,2}$, F. Blanco ${ }^{2}$, R. Meijide-Faílde ${ }^{1} .{ }^{1}$ Biological science, Medicine and Physiotherapy, University of A Coruña; ${ }^{2}$ Rheumatology, INIBICCHUAC, A Coruña, Spain

Background: A growing number of findings support the hypothesis that type 2 diabetes is an independent risk factor of osteoarthritis (OA). However, the mechanisms underlying the connexion between both diseases remain unclear. Hydro gen sulfide $(\mathrm{H} 2 \mathrm{~S})$ plays an important role in the pathogenesis of diabetes and its complications. In relation, we and other authors have observed a protective impact of $\mathrm{H} 2 \mathrm{~S}$ induction on activation of pathological pathways in the chondrocyte.

Objectives: In this study we examined the modulation of $\mathrm{H} 2 \mathrm{~S}$ levels in osteoarthritic chondrocytes from diabetic (DB) or non-diabetic (non-DB) patients subjected or under glucose stress, in order to elucidate whether impairment in H2Smediated signalling could participate in the establishment of diabetes-related $O A$. Methods: Chondrocytes were isolated from OA cartilage of diabetic (DB) or non diabetic (non-DB) patients. T/C28a2 and primary human chondrocytes were stimulated w/o IL-1 $\beta$ ( $5 \mathrm{ng} / \mathrm{mL})$ under a normal $(5.5 \mathrm{mM}$; NG) or a high $(25 \mathrm{mM}$; $\mathrm{HG}$ ) glucose environment. Gene and protein expression of enzymes involved in $\mathrm{H} 2 \mathrm{~S}$ synthesis (cystationine $\gamma$-liase [CSE], cystationine $\beta$-synthase [CBS], and 3 mercaptopyruvate sulfurtransferase [3-MT]) and $\mathrm{HO}-1$ were assessed by RTqPCR and WB, respectively. To determine the involvement of H2S in catabolic pathways activated by $\mathrm{HG}$ in chondrocytes, NaSH and GYY $4137(500 \mu \mathrm{M})$, a fast and slow-releasing $\mathrm{H} 2 \mathrm{~S}$ donor respectively, were employed.

Results: Fresh isolated chondrocytes from OA cartilage of diabetic patients showed lower levels of H2S synthesising enzymes (CSE, CBS and 3-MT) than those of non-DB patients (figure 1). In relation, chondrocytes T/C28a2 exposed to HG stress expressed lower mRNA and protein levels of these 3 enzymes after 3 days of incubation compared to those incubated in NG conditions $(0.41$-fold and 0.83-fold [CSE], 0.42-fold and 0.66-fold [CBS], and 0.52-fold and 0.79-fold [3-MT] for mRNA and protein expression, respectively; $n=6, p<0.05)$. IL-1 $\beta$ also attenuated the gene and protein expression of CBS elicited by chondrocytes incubated in NG (0.47-fold and 0.86-fold, respectively; $n=6, p<0.05)$. Additionally, the expression of pro-inflammatory chemokine IL-8 induced by IL-1 $\beta$ was significantly higher in chondrocytes under HG than NG condition (6-fold; $n=5, p<0.05$ ) whereas protein levels of heme oxygenase 1, an anti-inflammatory enzyme, were reduced in $\mathrm{HG}$ exposed chondrocytes (0.77-fold; $\mathrm{n}=6, \mathrm{p}<0.05)$. GYY 4137 and $\mathrm{NaSH}$ co-treatment recovered HO-1 expression and reduced IL-8 levels in chondrocytes under IL-1 $\beta+\mathrm{HG}$ conditions. Furthermore, similar results were registered in primary human chondrocytes from OA cartilage. 